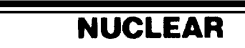

\title{
The status of normal conducting RF (NCRF) guns, a summary of the ERL2005 workshop
}

\author{
David H. Dowell ${ }^{\mathrm{a}, *}$, John W. Lewellen ${ }^{\mathrm{b}}$, Dinh Nguyen ${ }^{\mathrm{c}}$, Robert Rimmer ${ }^{\mathrm{d}}$ \\ ${ }^{a}$ Stanford Linear Accelerator Center, 2575 Sand Hill Road, Menlo Park, CA 94025, USA \\ b Argonne National Laboratory, 9700 S. Cass Ave., Argonne, IL 60439, USA \\ ${ }^{\mathrm{c}}$ Los Alamos National Laboratory, P.O. Box 1663, Los Alamos, NM 87545, USA \\ d Jefferson Laboratory, 12000 Jefferson Avenue, Newport News, VA 23606, USA
}

Available online 21 November 2005

\begin{abstract}
The 32nd Advanced ICFA Beam Dynamics Workshop on Energy Recovering Linacs (ERL2005) was held at Jefferson Laboratory, March 20-23, 2005. A wide range of ERL-related topics were presented and discussed in several working groups with Working Group 1 concentrating upon the physics and technology issues for DC, superconducting RF (SRF) and normal conducting RF (NCRF) guns. This paper summarizes the NCRF gun talks and reviews the status of NCRF gun technology. It begins with the presentations made on the subject of low-frequency, high-duty factor guns most appropriate for ERLs. One such gun at $433 \mathrm{MHz}$ was demonstrated at $25 \% \mathrm{DF}$ in 1992, while the CW and much improved version is currently being constructed at $700 \mathrm{MHz}$ for LANL. In addition, the idea of combining the NCRF gun with a SRF linac booster was presented and is described in this paper. There was also a talk on high-field guns typically used for SASE-free electron lasers. In particular, the DESY coaxial RF feed design provides rotationally symmetric RF fields and greater flexibility in the placement of the focusing magnetic field. While in the LCLS approach, the symmetric fields are obtained with a dual RF feed and racetrack cell shape. Although these guns cannot be operated at high-duty factor, they do produce the best quality beams. With these limitations in mind, a section with material not presented at the workshop has been included in the paper. This work describes a re-entrant approach which may allow NCRF guns to operate with simultaneously increased RF fields and duty factors. And finally, a novel proposal describing a high-duty factor, two-frequency RF gun using a field emission source instead of a laser driven photocathode was also presented.
\end{abstract}

(C) 2005 Elsevier B.V. All rights reserved.

PACS: 29.27.Bd; 41.60.Cr; 52.59.-f; 41.75.-I; 29.27.Ac; 41.60.Ap

Keywords: RF guns; Normal conducting; Superconducting; Radio frequency fields

\section{Introduction (D. H. Dowell, SLAC)}

This report summarizes the normal conducting RF (NCRF) gun presentations given in the Working Group I session at the Jefferson Lab ERL2005 Workshop. Although the re-entrant gun material of Section 3 was not presented in the workshop, we consider it to be very relevant to this topic and include it in this summary.

The report is organized into the following sections: Section 2 reviews the best-demonstrated performance for a NCRF gun at high-duty factor. Next Dinh Nguyen

\footnotetext{
*Corresponding author. Tel.: 6509262494 ; fax: 6509268533.

E-mail address: dowell@slac.stanford.edu (D.H. Dowell).
}

describes a next-generation NCRF gun LANL and AES have designed for $\mathrm{CW}$ operation. This $700 \mathrm{MHz}$ gun is scheduled for completion in 2006 and should surpass many of the performance parameters of any previous NCRF system. Dinh also proposes combining the NCRF gun with a SRF booster linac for a hybrid injector design. In Section 4 Robert Rimmer applies experience gained in building the PEP-II cavities for the B-Factory at SLAC to propose a more efficient NCRF design. In his approach the cells are made with very re-entrant nose cones to increase the onaxis RF field, improve the shunt impedance and reduce the thermal stresses. Although this work was not presented in the working group, it has been included because of its relevance to the discussion of NCRF guns. Coaxial fed 
guns are described in Section 5. The principle advantages of this type of gun are the elimination of all RF field asymmetries and complete freedom in the placement of the emittance compensation solenoid. This gun has produced excellent quality beams for the VUV-FEL at DESY in Hamburg, Germany. Another approach to generating symmetric fields is given in Section 6. In this gun design for the SLAC/LCLS X-ray FEL, a rotationally symmetric field is achieved with dual RF feeds and an elliptical cavity shape. In Section 7, John Lewellen proposes a twofrequency gun to limit the time for field emission from a cathode stalk to a small portion of an RF cycle. In this way he eliminates the need for a drive laser. Section 8 is the summary.

\section{State-of-the-art (D. H. Dowell, SLAC)}

In terms of duty factor and average power operation, the Boeing/LANL $433 \mathrm{MHz}$ gun remains the state-of-the-art. This gun was fabricated in 1988-1989 [1] and tested at high-average power from 1990-1992 up to $5 \mathrm{MeV}$ beam energy [2]. In 1994-1996 it was incorporated into a higher energy accelerator $(20 \mathrm{MeV})$ and was used as the electron source in bunch compression experiments demonstrating third-harmonic linearization of the longitudinal phase space [3]. Fig. 1 shows a photograph taken from the photocathode end of the gun. The cathode deposition chamber is in the foreground with the large RF waveguide feeds (black) connected at $45^{\circ}$ (relative to vertical) to independently power the two gun cells.

Table I lists the parameters for the $433 \mathrm{MHz}$ gun, which were demonstrated during the 1992 high-duty test. Unfortunately, the gun's performance was severely limited by poor vacuum which reduced the cathode lifetime to only $2-3 \mathrm{~h} \mathrm{[2].} \mathrm{This} \mathrm{short} \mathrm{lifetime} \mathrm{was} \mathrm{due} \mathrm{to} \mathrm{a} \mathrm{vacuum} \mathrm{leak} \mathrm{from}$ the water-cooling channels into the gun as evidenced by the large partial pressure of water.

The general configuration of this gun is given in Fig. 2. In this design, the emittance compensation coil is embedded (brazed) into the copper structure. This brazing distorted the embedded coil producing a large dipole kick. This dipole magnetic field was corrected using four permanent magnets clocked at $90^{\circ}$ around the coil [4]. After correcting for the distortion, the rms emittance was measured to be fairly good at $[4.4+1.1 * \mathrm{Q}(\mathrm{nC})]$ microns. The general shape of the gun cells can also be seen in Fig. 2. The re-entrant design allowed the relatively high rf field of $25 \mathrm{MV} / \mathrm{m}$ (peak) to be reached at high-duty factor. This same re-entrant shape for the nose cones is further optimized to reduce thermal stress as described in Section 4.

\section{The Los Alamos/AES CW NCRF gun (D. Nguyen, LANL)}

A key component of an energy recovery linac is a lowemittance, high-average-current electron gun. The electron beam's average current $(\sim 1 \mathrm{~A})$ determines the electron bunch charge $(\sim 1 \mathrm{nC})$ and bunch repetition rate $(\sim 1 \mathrm{GHz})$. The most straightforward approach to achieve low emittance ( $\sim 2 \mathrm{~mm}$-mrad) at $1 \mathrm{nC}$ bunch charge is through the use of the room-temperature RF photocathode gun. These qualities have been achieved with RF guns operating at low-duty factors. To date, a high-average-current RF photocathode gun, operating continuously at $100 \%$ duty factor, is yet to be demonstrated. The principal challenges of a high-duty-factor normal-conducting RF gun are

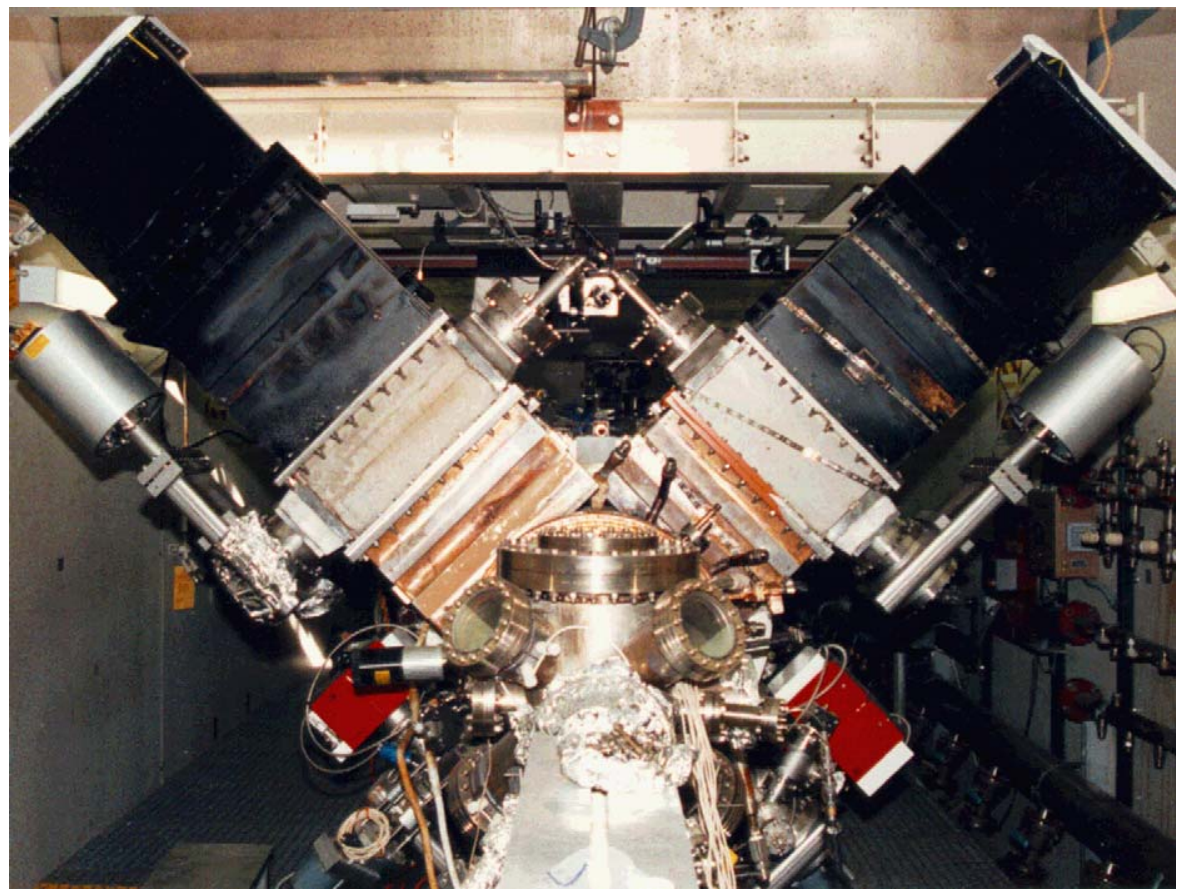

Fig. 1. Photograph of the Boeing/LANL $433 \mathrm{MHz}$ NCRF gun in the test vault. 
caused by the large RF ohmic losses in the cavity walls and the relatively poor vacuum environment of the RF injectors under continuous operation. What is needed is a demonstration of thermal and vacuum management of a room-temperature RF gun operating continuously ( $100 \%$ duty).

Table 1

Parameters demonstrated during the 1992 high-duty test of the $433 \mathrm{MHz}$ NCRF gun

\begin{tabular}{ll}
\hline Photocathode performance: & \\
Photosensitive material: & $\mathrm{K}_{2} \mathrm{CsSb}$ Multialkali \\
Quantum efficiency: & $5-12 \%$ \\
Peak current: & $45-132 \mathrm{~A}$ \\
Cathode lifetime: & $1-10 \mathrm{~h}$ \\
Angle of incidence: & Near-normal incidence \\
Gun parameters: & \\
Cathode gradient: & $26 \mathrm{MV} / \mathrm{m}$ \\
Cavity type: & Water-cooled copper \\
Number of cells: & 4 \\
RF frequency: & $433 \times 10^{6} \mathrm{Hertz}$ \\
Final energy: & $5 \mathrm{MeV}(4-\mathrm{cells})$ \\
RF power: & $600 \times 10^{3} \mathrm{Watts}$ \\
Duty factor: & $25 \%, 30 \mathrm{~Hz}$ and $8.3 \mathrm{~ms}$ \\
Laser parameters: & \\
Micropulse length: & $53 \mathrm{ps}, \mathrm{FWHM}$ \\
Micropulse frequency: & $27 \times 10^{6} \mathrm{~Hz}$ \\
Macropulse length: & $10 \mathrm{~ms}$ \\
Macropulse frequency: & $30 \mathrm{~Hz}$ \\
Wavelength: & $527 \mathrm{~nm}$ \\
Cathode spot size: & $3-5 \mathrm{~mm} \mathrm{FWHM}$ \\
Temporal and transverse & \\
distribution: & $\mathrm{Gaussian}, \mathrm{Gaussian}$ \\
Micropulse energy: & $0.47 \mu \mathrm{J}$ \\
Energy stability: & $1-5 \%$ \\
Pulse-to-pulse separation: & $37 \mathrm{~ns}$ \\
Micropulse frequency: & $27 \times 10^{6} \mathrm{~Hz}$ \\
Gun performance: & \\
Emittance ( $\mu \mathrm{m}, \mathrm{RMS}):$ & $5-10 \mathrm{for} 1-7 \mathrm{nC}$ \\
Charge: & $1-7 \mathrm{nC}$ \\
Energy: & $5 \mathrm{MeV}$ \\
Energy spread: & $100-150 \mathrm{keV}$ \\
\hline & \\
&
\end{tabular}

Funded by NAVSEA and the JTO, Los Alamos and Advanced Energy Systems (AES) have designed a watercooled $700 \mathrm{MHz}$ copper photocathode gun with a dense array of cooling channels for thermal management and sufficient vacuum pumping to provide a good vacuum in the photocathode cell [5]. The design of a normalconducting, $700 \mathrm{MHz}$ gun operating at 7,7 and $5 \mathrm{MV} / \mathrm{m}$ is shown in Fig. 3. The photocathode gun is designed to produce $2.5 \mathrm{MeV}$ electron beams. It consists of a $\pi$-mode, $2 \frac{1}{2}$-cell, RF cavity with on-axis electric coupling and emittance compensation, and a non-resonant vacuum plenum. The non-resonant vacuum plenum can accommodate up to eight ion pumps to ensure adequate vacuum pumping of the RF injector. Large-diameter apertures between the resonant cells and the non-resonant vacuum plenum are used to maintain high-conductance passages for pumping the photocathode cell. Heat removal in the resonant cells is achieved via dense arrays of internal cooling passages capable of handling high-velocity water flows. The septum walls are almost flat to keep the cooling channels as close to the RF surface as possible. Megawatt RF power is coupled into the gun through two tapered ridge-loaded waveguides [6]. PARMELA simulations show that the room-temperature RF photocathode gun can produce a $6 \mathrm{~mm}$-mrad emittance at $3 \mathrm{nC}$ bunch charge.

The $2 \frac{1}{2}$-cell, RF gun is being fabricated at AES with an expected delivery of January 2006. Concurrent efforts are being carried out to prepare the Low-Energy Accelerator Facility (LEDA) for the installation, commissioning and testing the room-temperature RF gun. As soon as the NCRF gun is delivered to Los Alamos, we plan to perform the thermal test with a $1 \mathrm{MW} 700 \mathrm{MHz}$ klystron in the LEDA facility. This thermal test will answer the question whether sufficient gradient can be applied continuously to the normal-conducting $\mathrm{RF}$ gun to pull up to $3 \mathrm{nC}$ of electron charge per bunch and still maintain thermomechanical integrity in the RF gun. The thermal test is expected to complete by mid-2006. Subsequent beam tests using either the bi-alkali $\left(\mathrm{K}_{2} \mathrm{CsSb}\right)$ or tri-alkali $\left(\mathrm{Cs}: \mathrm{Na}_{2} \mathrm{KSb}\right)$ as the photocathode are being planned and,

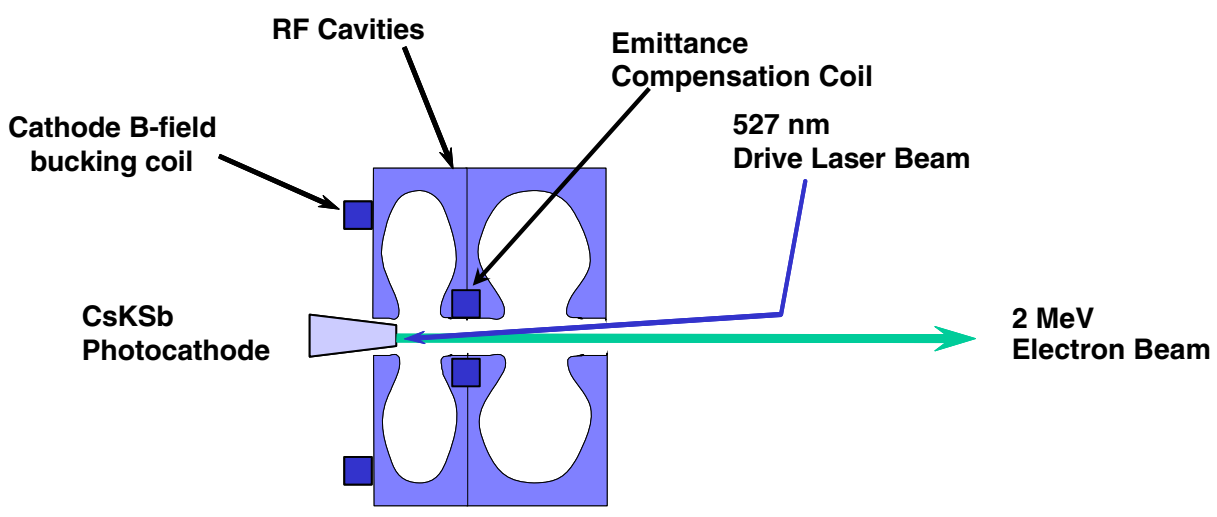

Fig. 2. Drawing of the $433 \mathrm{MHz}$ gun showing the re-entrant design and the locations of the emittance compensation coil and cathode field-bucking coil. Cells 3 and 4 indicated in Table 1 are not shown. The cathode was fabricated in an attached deposition chamber and inserted into the gun under vacuum via a long cathode stick. See Fig. 1 and Refs. [2,3]. 


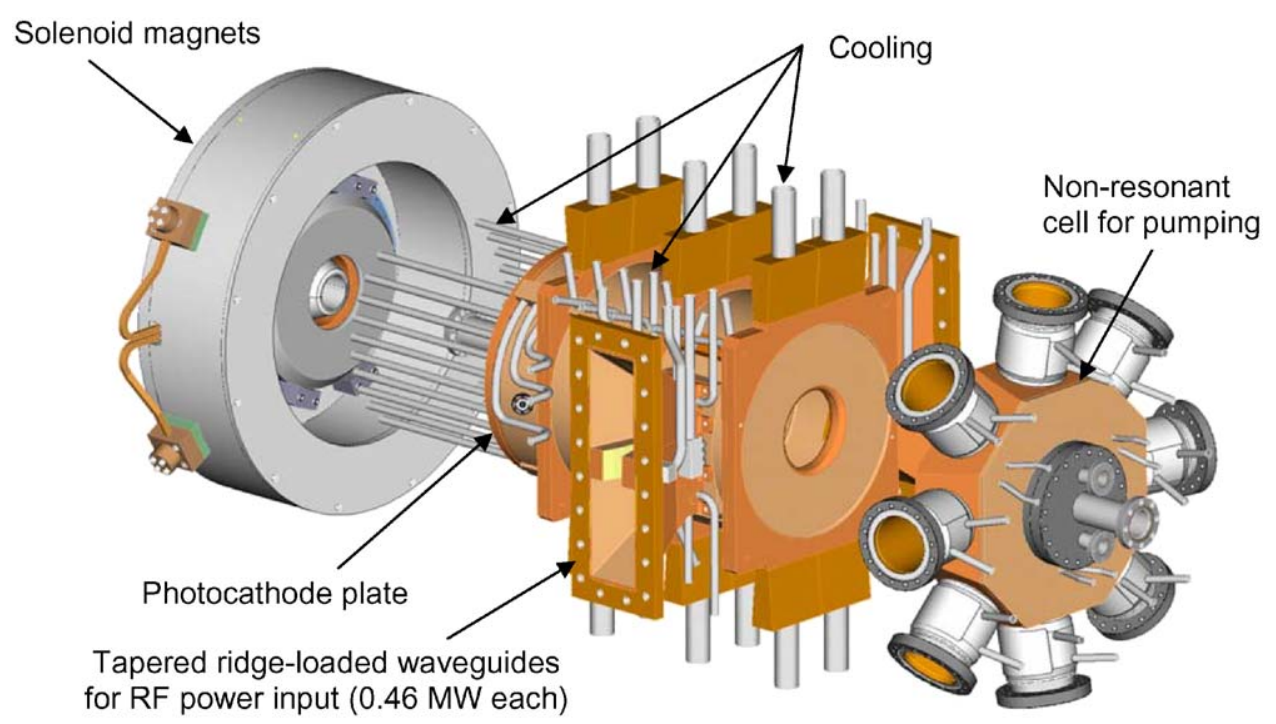

Fig. 3. Exploded view of the $2 \frac{1}{2}$ cell NCRF gun being fabricated by AES for LANL. The non-resonant cell provides additional vacuum pumping for the 1.5-cell gun.

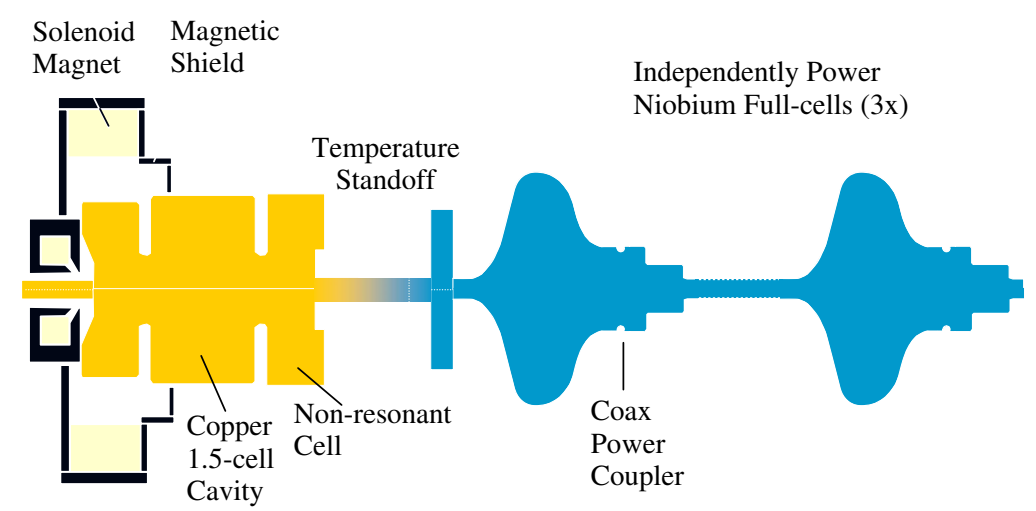

Fig. 4. A proposed hybrid NCRF gun + SRF booster injector based upon the LANL/AES NCRF gun.

if funding permits, scheduled for late 2006. This $2 \frac{1}{2}$-cell, $\mathrm{RF}$ photocathode gun can also be easily redesigned to suit the ERL applications.

A shorter version of this NCRF gun can be incorporated into a hybrid (normal-conducting + superconducting) RF photoinjector as shown in Fig. 4. This design consists of a normal-conducting, $1 \frac{1}{2}$-cell, RF gun followed by three independently driven RF superconducting cavities as energy boosters for the RF gun. Emittance compensation is accomplished through an external magnetic field near the photocathode. This magnetic field must be shielded from the superconducting cavities, as it is incompatible with superconductivity. High quantum-efficiency semiconductor photocathodes can be used at elevated temperatures in the first half-cell via a quarter-wave choke joint serving as thermal insulation. The cryogenically cooled interior provides an excellent vacuum that could significantly improve the photocathode lifetime. Release of debris from the photocathode into subsequent superconducting cavities, which could lead to quenching, can be minimized with a drift tube. The electron bunches generated in the normal- conducting $1 \frac{1}{2}$-cell RF gun are matched into independently powered superconducting RF cavities for acceleration to higher beam energy. RF power is coupled into the photoinjector cells from both sides via the APT-type RF power couplers that have been tested up to a few hundred kilowatts. In many high-current applications with significant beam loading, the loss of RF efficiency with use of normal conducting $1 \frac{1}{2}$-cell gun is negligible compared to the total system RF power consumption. The simplicity of a room-temperature RF gun outweighs any loss in RF efficiency when properly compared to the complexity of a fully superconducting gun.

\section{The LUX re-entrant gun (R. Rimmer, JLab)}

The goal of the Linac/Laser-Based Ultrafast X-ray Facility (LUX) normal-conducting photocathode gun proposed by Lawrence Berkeley National Laboratory is to combine the best of warm re-entrant cavity technology with the good properties of conventional laser photocathode sources. It is known from the long history of warm 
RF development that re-entrant cavities offer a significant advantage in efficiency over the traditional "pillbox" shape. For high-duty factor or $\mathrm{CW}$ sources this is very important, as these guns are most often limited by wall power dissipation rather than peak surface electric field. For a given stress level or surface power density a reentrant shape allows a higher electric field on the cathode. The re-entrant LUX design (see Fig. 5) shows that peak electric fields on the cathode of the order of $15 \mathrm{MV} / \mathrm{m}$ should be achievable in $\mathrm{CW}$ mode with a safety factor of 2 in stress, without resorting to exotic materials, cryogenic operation or other complications. The reasons for this are efficient RF design, a simple but effective cooling channel layout and a mechanical design that avoids stressconcentrating features like sharp corners and edges. The beam dynamics performance of the re-entrant gun is very similar to any other RF gun with comparable fields close to the axis.

The target cathode field for LUX was $64 \mathrm{MV} / \mathrm{m}$ for $5 \%$ duty factor at $1300 \mathrm{MHz}$, with an average power of $31 \mathrm{~kW}$ and a maximum wall-power density of about $100 \mathrm{~W} / \mathrm{sq}-\mathrm{cm}$ [7]. This equates to about $15.5 \mathrm{MV} / \mathrm{m} \mathrm{CW}$ with a peak surface temperature rise of $67^{\circ} \mathrm{C}$ and a maximum stress of $65 \mathrm{MPa}(\sim 9400 \mathrm{psi})$. While this level of stress is not insignificant it is well below the endurance limit of copper of $124 \mathrm{MPa}(\sim 18,000 \mathrm{psi})$ at 10,000 cycles [8] (equivalent to

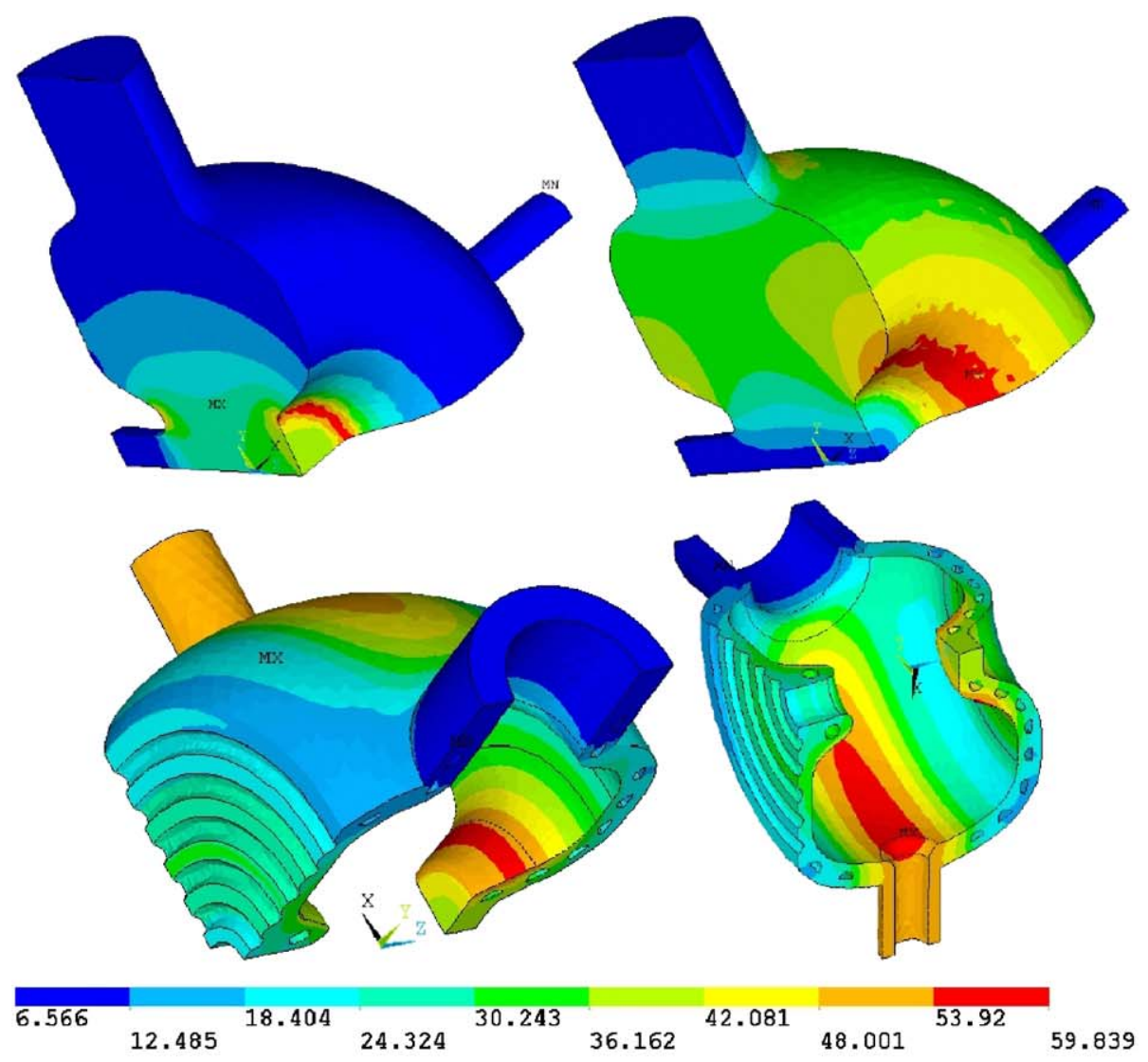

(a)
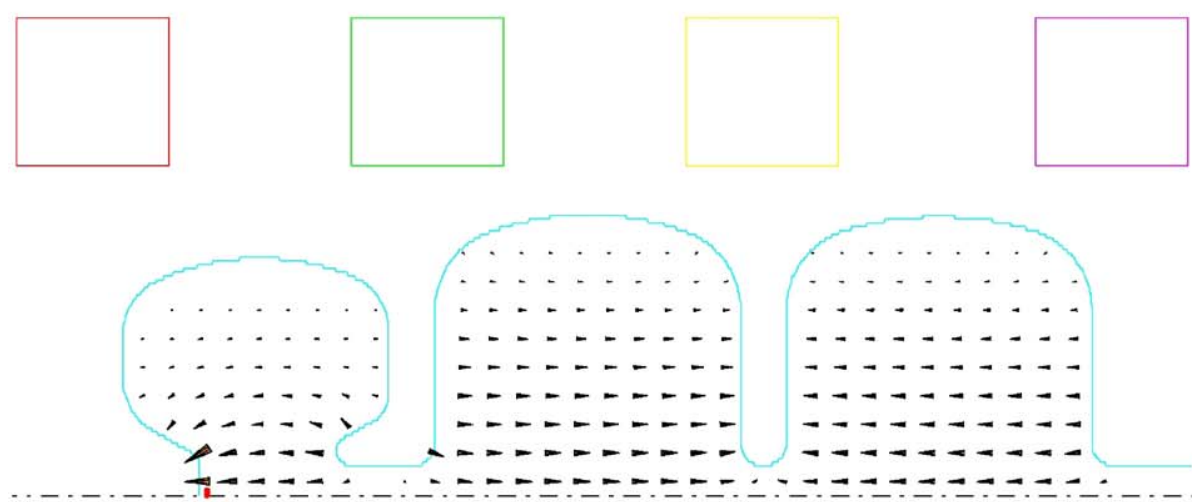

(b)

Fig. 5. Re-entrant cavity shape, RF field lines and placement of the solenoid coils of the LUX gun. 
three full-temperature cycles a day for 10 years). In fact, pushing the cavity to $20 \mathrm{MV} / \mathrm{m}$ only raises the stress to $108 \mathrm{MPa}$. For comparison, the PEP-II copper RF cavities were designed for $75 \mathrm{MPa}$ at $150 \mathrm{~kW}$ [9] and operate routinely at $\sim 100 \mathrm{~kW}$, and about $50 \mathrm{MPa}(\sim 7300 \mathrm{psi})$, with multi-ampere beam currents and hundreds of $\mathrm{kW}$ of $\mathrm{RF}$ power per cavity delivered to the beam. With careful design and careful stress management (such as varying the cooling channel density and routing the water to minimize thermal gradients) these stresses could be lowered further, allowing even higher cathode gradients.

Going to lower frequency also helps [10]. RF sources, couplers and windows operating at MW power levels are commonplace in L-band and should not be a limiting factor in gun design. Mechanical assembly does not have to be difficult or expensive if well though out, especially if the RF design is efficient to start with. Conventional cavity construction techniques within the accelerator and tube industries are more than adequate. Cutting openings into the cavity for pumping or RF power coupling can lead to local stress concentrations, but the LUX design shows that these can be held to levels at or below the peak stresses elsewhere in the body. Provided apertures are located symmetrically about the beam axis dipole kicks can be avoided. On-axis coupling would be another way to avoid the problem. While the LUX study focused on high-duty factor operation, the same shape optimization is valid for low repetition rate high-peak power guns, which are often limited by surface pulse heating. The efficient design minimizes surface magnetic fields, allowing higher-peak electric fields before the (magnetic) surface damage threshold is reached. This would be particularly useful for extreme guns such as those proposed for LCLS or the Tesla X-FEL.

\section{Coaxial feed NCRF guns (D.H. Dowell, SLAC)}

Implementing a coaxial $\mathrm{rf}$ power feed produces a rotationally symmetric $r f$ field. By moving the axial coupler to either the beam exit or through cathode stalk, the region along the cells is now available for placing the solenoid field anywhere along the length of the gun. The design for the Tesla Test Facility (TTF) [11] is shown in Fig. 6. In addition to the beam exit coupler approach, there is also a design for a high-duty factor NCRF gun with the rf coupling into the cavity through the cathode stick [10].

The complete freedom of unrestricted axial field shape along the gun allows for more ideal compensation matching of the bunch into the booster linac. Compensation for the various types of slice misalignment is done by matching the bunch transverse dynamics to the booster fields and acceleration.

\section{Symmetric dual-feed, race-track gun (D. H. Dowell, SLAC)}

The RF fields can also be made symmetric by using a dual feed and a race-track cell shape. The dual feeds cancel the dipole fields, and the elliptical cell shape corrects for the quadrupole field. The shape and integrated quadrupole kick, uncorrected and corrected, are shown in Fig. 7 for the LCLS gun [12].

\section{Field-emission cathode gating (J. W. Lewellen, ANL)}

A common feature of most high-brightness electron guns is the use of a photocathode drive laser to generate the electron beam. RF injectors, however, have been made using thermionic (hot) cathodes, which emit electrons simply due to their elevated temperature. Thermioniccathode rf (TCRF) guns have proven to be very successful sources of moderate quality (normalized emittances of $10-20 \mu \mathrm{m})$, moderate charge $(100-350 \mathrm{pC} /$ bunch $)$, highaverage macropulse current $(0.3-1 \mathrm{~A})$ beams.

The primary limiting aspect of TCRF gun performance is the continuous nature of the electron emission from the cathode. In fact, the main purpose of a photocathode drive

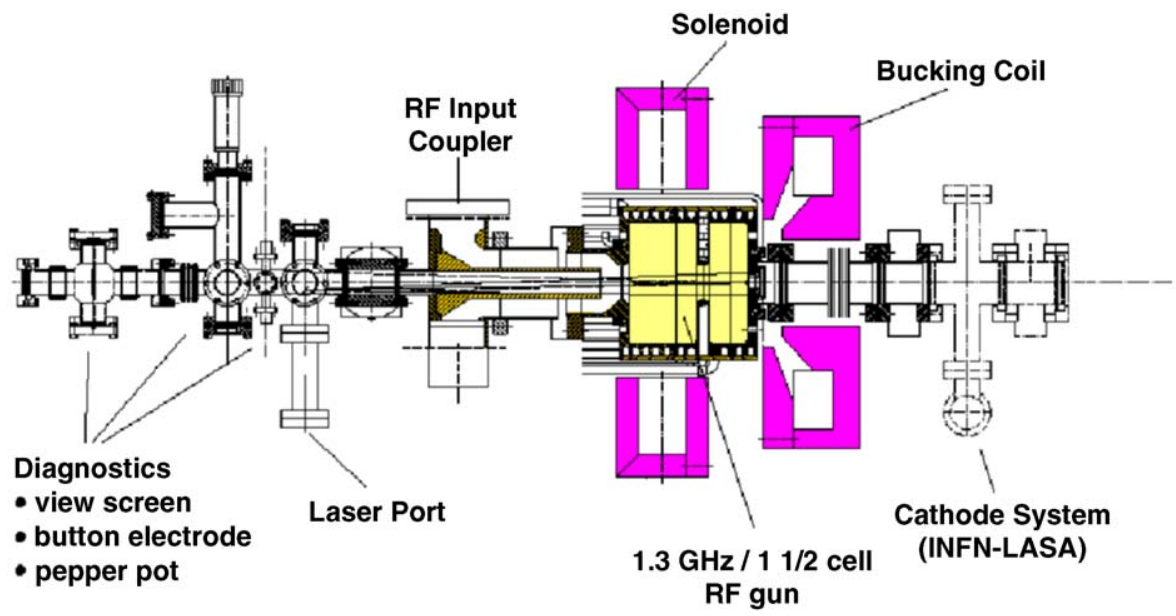

Fig. 6. Drawing of the Tesla Test Facility (TTF) NCRF gun with a coaxial rf feed. 

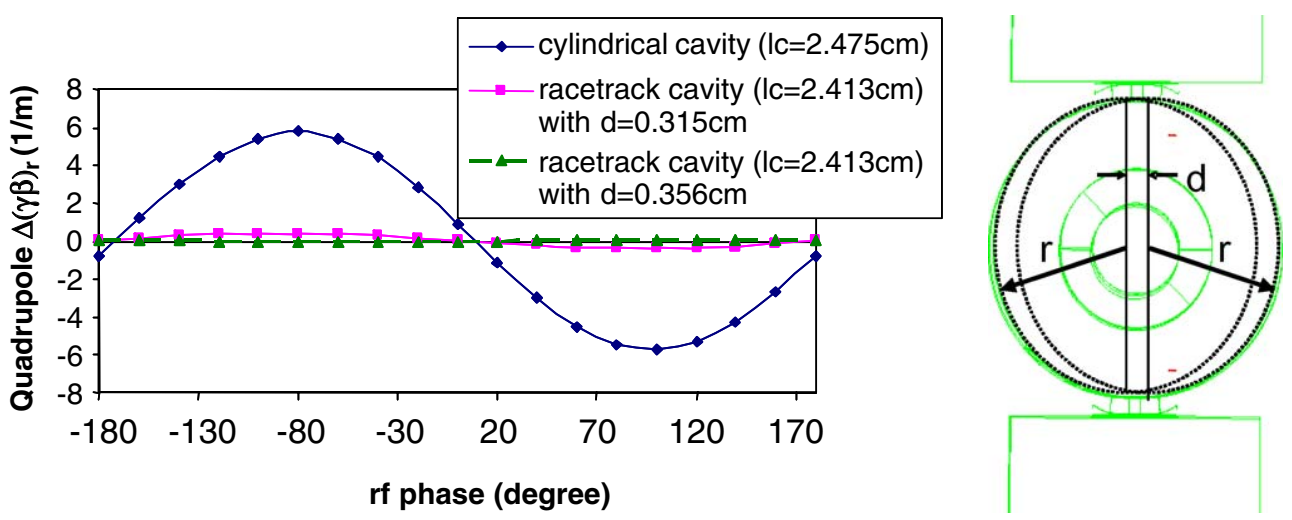

Fig. 7. The race-track shape and corrected and uncorrected quadrupole distortion for the LCLS gun design.

laser is to gate the electron emission to a small fraction of the rf period. The disadvantage of the photocathode gun is also embodied within the drive laser: high-average-current guns require high-power drive lasers as well as highquantum-efficiency photocathodes. The final result is that photocathode rf guns, as a rule, are far more complicated and expensive than thermionic-cathode rf guns.

There has recently been proposed [13] a method for gating electron emission from a field-emission (FE) cathode within an rf gun. By adding a third-harmonic component to the field within a gun cavity, the phase of peak gradient on the cathode is shifted from $90^{\circ}$. to approximately $50^{\circ}$. By carefully tuning the gun geometry (so as to control the ratio between the 1st and 3rd harmonics spatially as well as temporally), and by selecting the gradient and cathode parameters, the FE cathode emission window can be gated to approximately $10^{\circ}$. The method therefore combines the simplicity and potentially high-average currents of TCRF guns, with the narrow emission gating of photocathode guns.

The initial design was intended as a driver for highvoltage electron microscopes, and so is optimized for lowaverage currents $(0.1-10 \mathrm{~mA})$. In principle, however, the process can easily be extended to higher average beam currents; in such cases, the existing "bag of tricks" for dealing with large space-charge forces - such as solenoidal focusing, cathode-region focusing, etc. - can be brought to bear. Although much additional study and experimental verification remain, the method appears to be a promising approach towards development of high-current, highbrightness beam sources.

In principle, this technique could be applied to either NCRF or cryogenic rf guns; however, there is considerable appeal in terms of simplicity of design, making the FEcathode gating scheme a natural complement to a highduty-factor NCRF gun.

\section{Summary and conclusions (D.H. Dowell, SLAC)}

Working group presentations and discussions on the topic of NCRF guns fall into two general categories: those capable of operating at high-duty factor and those at low-duty factor. The high-duty factor guns have lower peak field on the cathode because of RF heating issues while the low-duty guns can have very high peak fields $(\sim 100 \mathrm{MV} / \mathrm{m})$ and thereby produce very bright beams. Many ERL applications require both high-duty factor and high beam brightness, necessitating significant efforts in thermal and vacuum engineering of the NCRF gun design for efficient heat removal and long cathode lifetime.

The high-power operation of a NCRF gun at $25 \%$ duty factor was first demonstrated in 1992. This $433 \mathrm{MHz}$ gun was a collaboration of Boeing and LANL. This gun is now 'retired'. A new $700 \mathrm{MHz}$ gun, currently under construction by AES for LANL, has been designed for true CW operation and is scheduled for completion in 2005. The high-power RF testing will be at LANL and will take advantage of the existing LEDA RF power and cooling infrastructure.

Low-duty factor NCRF guns represent the state-of-theart sources for high-quality electron beams. The guns developed for SLAC's LCLS and the DESY XFEL are examples of these types of high frequency (2.856 and $1.3 \mathrm{GHz}$, respectively) guns. In these guns the RF fields are rotationally symmetric and the cathode electric field is high $(\sim 60$ to $120 \mathrm{MV} / \mathrm{m})$. However, they are not capable of operating CW as needed for most ERL's.

Also presented in this working group was a reasonably high-average current $\mathrm{CW}$ gun which requires no drive laser. This two-frequency gun would use the field shaped by two RF frequencies to switch on and off a field-emission cathode.

The re-entrant gun described in this report suggests the possibility of NCRF guns with both high cathode electric fields $(\sim 20 \mathrm{MV} / \mathrm{m})$ and efficient $\mathrm{CW}$ operation. While not as efficient as the SRF gun, this approach when combined with a SRF booster linac could provide a technology bridge between the NCRF gun and the full SRF gun.

\section{Acknowledgements}

The work of David H. Dowell is supported by the US DOE Contract No. DE-AC03-76SF00515. The work of John W. Lewellen reported by this document is supported 
by US Department of Energy, Office of Basic Energy Sciences, under Contract No. W-31-109-ENG-38. The Normal-conducting RF Injector work at LANL and AES is supported by the DoD High Energy Joint Technology Office and NAVSEA. The Jefferson Laboratory work of R. Rimmer is funded by SURA, Inc., under Contract No. DE-AC05-84ER-40150 with the US Department of Energy. The LUX gun work was done at LBNL under contract DE-AC03-76SF00098.

\section{References}

[1] J.L. Warren, T.L. Buller, A.M. Vetter, Proceedings of the 1989 Particle Accelerator Conference.

[2] D.H. Dowell, K.J. Davis, K.D. Friddell, E.L. Tyson, C.A. Lancaster, L. Milliman, R.E. Rodenburg, T. Aas, M. Bemes, S.Z. Bethel, P.E. Johnson, K. Murphy, C. Whelen, G.E. Busch, D.K. Remelius, Appl. Phys. Lett. 63 (15) (1993) 2035;

D.H. Dowell, S.Z. Bethel, K.D. Friddell, Nucl. Instr. and Meth. A 356 (1995) 167.

[3] D.H. Dowell, J.L. Adamski, T.D. Hayward, P.E. Johnson, C.D. Parazzoli, A.M. Vetter, Nucl. Instr. and Meth. A 393 (1996) 184

[4] T. Hayward, private communication.
[5] D.C. Nguyen, et al., Nucl. Instr. Meth. Phys. Res. A 528 (2004) 71.

[6] S.S. Kurennoy, L.M. Young, Proceedings of the 2003 Particle Accelerator Conference, May 12-16, Portland, OR.

[7] Staples et al., EPAC 2004.

[8] R.P. Reed, R.P. Mikesell, Low-temperature mechanical properties of copper and selected copper alloys; a compilation from the literature, US Dept. of Commerce, National Bureau of Standards; for sale by the Superintendant of Documents, US Government Printing Office, 1967, p. 43.

[9] LBNL CBP Technical Note 197, LCC-0032 November 1999]

[10] R.A. Rimmer, A high-gradient CW RF photo-cathode electron gun for high current injectors, Proc. 2005 Particle Accelerator Conference, Knoxville, TN, 2005.

[11] F. Stephan, et al., Recent results and perspectives of the low emittance photo injector at PITZ, Proceedings of 2004 International Free Electron Laser Conference, Trieste, Italy, 2004, pp. $347-350$

[12] L. Xiao, et al., Dual feed rf gun design for LCLS, Proceedings of the 2005 Particle Accelerator Conference; Modifications on RF components in the LCLS injector; C. Limborg-Deprey, et al., Proceedings of the 2005 Particle Accelerator Conference; Z. Li, et al., Coupler design for the LCLS injector S-band structures, Proceedings of the 2005 Particle Accelerator Conference.

[13] J.W. Lewellen, J. Noonan, Field-emission cathode gating for $\mathrm{rf}$ electron guns, Phys. Rev. ST Accel. Beams 8 (2005) 033502. 